submitted to ApJL

\title{
Using Transit Timing Observations to Search for Trojans of Transiting Extrasolar Planets
}

\author{
Eric B. Ford ${ }^{1}$ and Matthew J. Holman \\ Harvard-Smithsonian Center for Astrophysics, Mail Stop 51, 60 Garden Street, Cambridge, \\ MA 02138 \\ eford, mholman@cfa.harvard.edu
}

\begin{abstract}
Theoretical studies predict that Trojans are likely a frequent byproduct of planet formation and evolution. We examine the sensitivity of transit timing observations for detecting Trojan companions to transiting extrasolar planets. We demonstrate that this method offers the potential to detect terrestrial-mass Trojans using existing ground-based observatories. We compare the transit timing variation (TTV) method with other techniques for detecting extrasolar Trojans and outline the future prospects for this method.
\end{abstract}

Subject headings: techniques: photometric — planetary systems: formation celestial mechanics

\section{Introduction}

For centuries, theories of planet formation had been designed to explain our own Solar System, but the first extrasolar planetary systems discovered were very different than our own (e.g., Mayor \& Queloz 1995). These discoveries led to the realization that planet formation theory must be generalized to explain a much greater diversity of planetary systems. Stable Trojan companions to extrasolar planets may be common. In our solar system, Mars, Jupiter, and Neptune each share their orbit with asteroids orbiting near the stable (L4/L5) Lagrange points that lead/trail the planet by $\simeq 60^{\circ}$. While the mass ratios of the Trojan

\footnotetext{
${ }^{1}$ Hubble Fellow
} 
systems in our solar system are rather extreme $\left(\leq 7 \times 10^{-9}\right)$, it is possible that extrasolar planets may have much more massive Trojans. Indeed, theorists have already outlined several possible mechanisms to form Trojans with mass ratios potentially including unity (Laughlin \& Chambers 2002; Chiang \& Lithwick 2005; Morbidelli et al. 2005; Thommes 2005; Cresswell \& Nelson 2006; Ford \& Gaudi 2006).

Trojans of both Jupiter and Neptune have provided clues about our own solar system's history (Michtchenko, Beauge \& Roig 2001; Kortenkamp, Malhotra \& Michtchenko 2003; Chiang \& Lithwick 2005; Morbidelli et al. 2005). Similarly, the detection of extrasolar Trojans would be useful for constraining theories of planet formation and migration. While all the above mechanisms predict that Trojans would survive the migration process, there are alternative models of planet migration that predict Trojans would not survive (Rasio \& Ford 1996; Wu \& Murray 2003; Gaudi 2003; Ford \& Rasio 2006; Ford \& Gaudi 2006). The detection of a Trojan companion to a short-period planet would present a serious challenge to these mechanisms for forming "hot Jupiters" and would imply that the planet in such a system was formed via migration through a dissipative disk rather than tidal circularization after approaching the star on a highly eccentric orbit. Thus, searching for extrasolar Trojans can test models of planet formation.

Previously, three methods have been proposed to identify extrasolar Trojans. If a Trojan is sufficiently massive and has a sufficiently large libration amplitude, then its presence could be inferred using the deviations from a Keplerian perturbation to the stellar radial velocity or astrometric signal caused by a single planet. Laughlin \& Chambers (2002) have shown that two comparable mass giant planets occupying a 1:1 mean motion resonance would typically have strong planet-planet gravitational interactions on a secular timescale. However, these signatures may not be unique: a reanalysis of the RV observations of HD 128311 and HD 82943 have shown that both of the current data sets are also consistent with a pair of planets in a 1:1 mean motion resonance (Gozdziewski \& Konacki 2006), as well as the originally published orbital solutions.

If a Trojan transits its parent star, then photometric or spectroscopic monitoring of stars with transiting planets (particularly at times offset from the planet transit by $\sim P / 6$ ) may reveal the Trojan transit via the decrease in stellar flux (Rowe et al. 2000) or anomalous RV excursions due to the Rossiter-McLaughlin effect (Gaudi \& Winn 2006). Both these methods are more sensitive to large Trojans. While ground-based observations are not sensitive to Earth-sized planets, space observations could detect such Trojans. However, it is not guaranteed that a Trojan will transit its parent star, as it may have a significant inclination (e.g., Morbidelli et al. 2005). Further, since the libration period can be quite large, long-term monitoring would be required to ensure detection. 
Ford \& Gaudi (2006) proposed a method for detecting a Trojan companion based on combining radial velocity observations and photometric observations of a transiting planet. Even if the Trojan itself were not transiting, it could reveal it's presence via a time lag between the radial velocity null and the time of central transit. Existing observations already place significant (99.9\%) upper limits on the mass of Trojan companions to HD 209458b and $\mathrm{HD} 149026 \mathrm{~b}$ of $\simeq 13 M_{\oplus}$ and $\simeq 25 M_{\oplus}$.

Here, we present another method for detecting Trojan companions to extrasolar planets using only photometric observations of transiting extrasolar planets. Once a transiting planet has been identified, higher precision follow-up observations and modeling can precisely measure the mid-time of subsequent transits (currently with a precision $\sim 10 \mathrm{~s}$; e.g., Agol \& Steffen 2006; Holman et al. 2006; Winn et al. 2006). If the star and the transiting giant planet are the only bodies in the system, then the transits will be strictly periodic, i.e., $t_{i}=t_{0}+i \times P_{s}+\delta t_{i}$, where $t_{i}$ is the time of the $i$ th transit, $P_{s}$ is the transiting planet's sidereal orbital period, and any transit timing variations $\left(\delta t_{i}\right)$ are due to measurement error. However, if an additional planet orbits the star, then the times of the giant planet's transits will be affected (Miralda-Escude 2002; Holman \& Murray 2005; Agol et al. 2006; Heyl \& Gladman 2006). By analyzing the deviations of the observed TTVs from a strictly periodic model $\left(\delta t_{i}\right)$, astronomers can search for additional planets orbiting the star. Here, we show that a sub-Earth mass Trojan planet could induce a transit timing signal that is easily measurable using existing ground-based observatories.

\section{Observational Constraints on Trojans}

We consider a three body system and denote the stellar mass $\left(m_{\star}\right)$, the planet mass $\left(m_{p}\right)$, and the Trojan mass $\left(m_{T}\right)$. We refer to all bodies librating about the L4 or L5 fixed point of a planet as "Trojans". If there are no other massive bodies in the system, then the L4/L5 fixed points are stable for circular orbits if the ratio, $\mu=\left(m_{p}+m_{T}\right) /\left(m_{\star}+m_{p}+m_{T}\right)$, is less than a critical threshold $\mu_{c}$, where $0.03812 \leq \mu_{c} \leq 0.03852$ and $\mu_{c}$ depends on the ratio, $\epsilon \equiv m_{T} /\left(m_{p}+m_{T}\right)$ (Laughlin \& Chambers 2002). If the planet and Trojan have equal eccentricities and the Trojan resides exactly at the L4/L5 fixed point, then the transit timing signature for the primary planet would be indistinguishable from a similar system without a Trojan. More generally, for a Trojan on an orbit that is librating about the L4/L5 fixed point, the times of the primary planet's transits will deviate from being strictly periodic. Here, we focus our attention on Trojans that undergo small librations about the L4/L5 fixed points and are significantly less massive than the currently known planet.

The libration can be approximated as a linear superposition of two epicyclic motions. 
The star-Trojan separation can oscillate about the semi-major axis of the planet $\left(a_{p}\right)$ with the amplitude, $\delta a \ll \mu^{1 / 2} a_{p}$ on a timescale $\tau_{\text {fast }} \simeq P_{s}$, and the guiding center of the Trojan can oscillate on a longer timescale, $\tau_{\text {slow }} \simeq P_{s} \sqrt{27 /(4 \mu)}$ (Murray \& Dermott 2000). The timescale of the libration of the guiding center makes this motion most readily detectible by transit timing observations.

For a transiting planet, both $P_{s}$ and each $t_{i}$ can be measured precisely using photometry alone. Considering a series of continuous photometric observations with uncorrelated Gaussian uncertainties of magnitude $\sigma_{p h}$, taken at a rate $\Gamma$ around a single transit, the mid-transit time can be measured with an accuracy of $\sigma_{t_{i}} \simeq \sqrt{t_{e} / 2 \Gamma} \sigma_{p h} \rho^{-2}$, where $t_{e}$ is the duration of ingress/egress and $\rho$ is the ratio of the planet radius to stellar radius (Ford $\&$ Gaudi 2006). For typical parameters (e.g., $\sigma_{p h} \sim 10^{-3}$ ), $t_{i}$ can be measured to $\simeq 10 \mathrm{~s}$ (e.g., Brown et al. 2001; Holman et al. 2006). The period can be measured much more accurately than $t_{i}$, from observations of multiple transits separated by many orbits. For small amplitude libration about L4/L5 and circular orbits, the transit timing perturbation is given by $\delta t_{i} \simeq \epsilon P_{s} \Delta M\left(t_{i}\right) /(2 \pi)$, where $\Delta M\left(t_{i}\right)$ is the angular displacement of the Trojan from L4/L5 at the time of the $i$ th transit. The TTVs can be modeled by a sinusoid, $\delta t_{i}=K_{\mathrm{tt}} \sin \left(2 \pi\left(t-t_{0}\right) / P_{\mathrm{TTV}}+\phi\right)$, where $K_{\mathrm{tt}}$ is the amplitude of the transit timing variations and $P_{\mathrm{TTV}} \sim \tau_{\text {slow }}$. If the dominant periodicity of the transit timing variations $\left(P_{\mathrm{TTV}}\right)$ is well determined, then the remaining parameters can be determined via linear least squares fitting to the observed transit times. The transit timing variations will have an amplitude

$$
K_{\mathrm{tt}} \simeq 60 s\left(\frac{P}{4 d}\right)\left(\frac{m_{T}}{m_{\oplus}}\right)\left(\frac{0.5 M_{\mathrm{Jup}}}{m_{p}+m_{T}}\right)\left(\frac{K_{\Delta M}}{10^{\circ}}\right),
$$

where $K_{\Delta M}$ is the amplitude of the Trojan's angular displacement from the Lagrange point. For small amplitude libration, $K_{\Delta M} \simeq \max |\Delta M|$ and $\operatorname{rms}\left(\delta t_{i}\right) \simeq K_{\mathrm{tt}} / \sqrt{2}$ (see Fig. 1) Libration amplitudes of $K_{\Delta M} \sim 5-25^{\circ}$ are common for Trojans orbiting near the SunJupiter Lagrange points (Murray \& Dermott 2000).

The Lomb-Scargle periodogram can be easily adapted to efficiently scan a range of putative periods and identify any significant periodicities (Cumming 2004). If we assume that there are many $\left(N_{\mathrm{tt}}\right)$ transit timing observations with uncorrelated Gaussian uncertainties $\sigma_{t_{i}}=\sigma_{t t}$, that the transit timing observations are evenly distributed, and the duration of observations $\left(T_{\mathrm{obs}}\right)$ is greater than than $P_{\mathrm{TTV}}$, then a periodogram-style analysis results in a $50 \%$ chance of detecting a Trojan if $K_{t t} \geq K_{1 / 2} \simeq \sigma_{t t}\left(\frac{4}{N_{\mathrm{tt}}} \log \left[T_{\mathrm{obs}} /\left(2 F P_{s}\right)\right]\right)^{1 / 2}$ (Cumming 2004 ), where $F$ is the false alarm probability, which we set to $10^{-3}$. For $N_{\mathrm{tt}}=T_{\mathrm{obs}} / P_{\mathrm{s}}=40$, $K_{1 / 2} \simeq \sigma_{t t}$, so sub-Earth-mass Trojans could be readily detected. We note that all published transit timing data sets have $N_{\mathrm{tt}}<20$, which results in a significantly reduced sensitvity, if $P_{\mathrm{TTV}}$ is unknown a priori. In this small- $N_{\mathrm{tt}}$ regime, a simple $\chi^{2}$ test of the null hypothesis 
$\left(\delta t_{i}=0\right)$ is more sensitive for detecting transit timing variations. However, if only a single periodicity (e.g., $\tau_{\text {slow }}$ ) is to be tested, then even a modest number of observations can be quite sensitive (e.g., $K_{1 / 2} \simeq 2.5 \sigma_{\mathrm{tt}}$ even for $N_{\mathrm{tt}}=13$ ).

Once a Trojan has been detected, a Fisher information analysis (e.g., Gaudi \& Winn 2006) reveals that the uncertainty in $K_{\mathrm{tt}}$ will approach $\sigma_{K_{\mathrm{tt}}}=\sqrt{4 / N_{t t}} \sigma_{t t}$. If a Trojan were present, then the uncertainty in $K_{\mathrm{tt}}$ would set the uncertainty in the measurement of the mass of the Trojan to be

$$
\frac{\sigma_{m_{T}}}{m_{\oplus}} \simeq \frac{0.5}{\sqrt{N_{\mathrm{tt}}}}\left(\frac{\sigma_{\mathrm{tt}}}{10 s}\right)\left(\frac{4 d}{P}\right)\left(\frac{m_{p}}{0.5 M_{\mathrm{Jup}}}\right)\left(\frac{10^{\circ}}{K_{\Delta M}}\right)
$$

Thus, transit timing observations can be very sensitive to sub-Earth-mass Trojan companions. However, due to the degeneracy between $m_{T}$ and $K_{\Delta M}$, the amplitude of transit timing detections due to a Trojan would not provide a strong upper limit on the Trojan mass. For Trojans with significant libration amplitudes, this degeneracy could be resolved by combining the amplitude with the measured $P_{\text {TTV }}$ (see Fig. 1 center). For Trojans with small libration amplitudes, the measured $P_{\mathrm{TTV}}$ will provide an upper limit for the libration amplitude and hence a lower limit to the Trojan-planet mass ratio. Regardless, the transit timing observations could be used to predict the time of transit of a Trojan and targeted photometric or spectroscopic follow-up observations could place an upper limit on the radius of the Trojan (e.g., Rowe et al. 2006; Gaudi \& Winn 2006). Combining such observations with planetary structure models (e.g., Valencia et al. 2007) could provide an upper limit on the Trojan mass and hence a lower limit for the libration amplitude.

\section{Discussion}

One long-term goal of immense scientific and public interest is to discover and study rocky planets, and eventually terrestrial planets that have masses, sizes, orbits, atmospheres, and perhaps even surface conditions similar to those of the Earth. Previous studies have demonstrated that the magnitude and timescale of transit timing variations due to Earthmass planets are readily detectable if they orbit near an interior or exterior low-order mean motion resonance (Holman \& Murray 2005; Agol et al. 2005). The TTV method is quite sensitive to planets near resonances (e.g., the planetary systems GJ 876, HD 128311, HD 73526, 55 Cnc, and HD 82943; Butler et al. 2006) that are particularly valuable for studying orbital dynamics and planet formation (Lee \& Peale 2002) and challenging for radial velocity and astrometric searches. The TTV method would also be able to confirm some planet candidates (likely to be identified by future transit searches) by detecting the orbital interactions of the planets, similar to the methods used for confirming the planets around PSR $1257+12$ 
(Rasio 1992; Malhotra 1993) and PSR1620-26 (Ford et al. 2000). This could prove particularly valuable for planet candidates that have small masses and/or orbit faint stars, so that radial velocity confirmation is impractical (e.g., most of the 16 transiting planet candidates orbiting faint stars recently published by Sahu et al. 2006, and the many transiting planets expected to be found by future space missions).

It would be extremely exciting to detect a transiting Earth-mass planet. Such a detection would enable follow-up observations to study the physical properties of the planet, such as the planet's radius and density (Brown et al. 2001, Sato et al. 2005, Charbonneau et al. 2006), the atmospheric composition (Charbonneau et al. 2002, Deming et al. 2005, Bozorgnia et al. 2006), and possibly even "resolve" surface/atmospheric features (Ford et al. 2001; Harrington et al. 2006; Gaidos et al. 2006).

We have demonstrated that a sub-Earth-mass Trojan planet could also result in a transit timing signal that can be readily measured with ground based observatories. Since the orbital planes are likely nearly aligned, the fact that a giant planet is already known to transit the star increases the odds that other planets orbiting that star will also transit (Holman \& Murray 2005). Thus, the transit timing method is particularly good at searching for transiting Earth-like Trojan planets that would enable extremely interesting follow-up observations. Our technique could be applied to search for terrestrial-mass Trojans of giant planets orbiting in the habitable zone of their stars (Schwarz et al. 2005), particularly for low mass stars where the habitable zone can be $\simeq 0.015 \mathrm{AU}$ away from the star. Once transitting terrestrial mass planets are discovered, this technique could be extended to search for extrasolar Trojans with asteroid-like masses.

While $\S 2$ and previous work have emphasized the sensitivity of transit timing observations, we caution that solving the inverse problem of determing planet properties from transit timing observations is likely to pose a significant challenge and be more difficult than interpreting other types of extrasolar planet observations. For example, in the radial velocity method, the dominant periodicity in the observed time series is readily identified with the orbital period of a massive companion and the amplitude of the variations is proportional to the mass of the companion (Konacki \& Maciejewski 1999). However, in TTV data, the dominant periodicity could be due to any one of several physical effects (see Fig. 2), including the reflex motion of the star due to the second planet (with a period equal to the orbital period of the second planet), the long-term mutual gravitational perturbations between the planets (with a period much longer than either orbital period), the short-term gravitational perturbations on the orbit of the transiting giant planet (on an intermediate timescale), or the light travel time due to a distant companion (e.g., Borkovitz et al. 2003; Heyl \& Gladman 2006). Therefore, even once a periodicity has been identified, it is not obvious what physical 
effect is causing the periodicity. Further, TTV signatures are more complex than the signatures of other dynamical detection techniques. For example, radial velocity observations of a multiple planet system can often be modeled by the linear superposition of multiple Keplerian orbits (Butler et al. 2006; Ford et al. 2006). However, for transit timing observations, the signal is often dominated by the deviations from such a simplified model. Therefore, it is necessary to perform n-body simulations to accurately calculate the TTV signature of each possible model (Holman \& Murray 2005; Agol et al. 2005; Steffen \& Agol 2005). Given the computational requirements of each n-body integration, practical algorithms must explore the high-dimensional $\left(\simeq 7 \times N_{p l}\right.$ ) parameter space very efficiently and rapidly converge on all physical models consistent with the observations.

A Trojan companion with small libration amplitude will induce a TTV signature that can be well approximated by a single sinusoid (Fig. 2, top). This contrasts with the TTV perturbations due to a planet near a different resonance (Fig. 2, middle and bottom). A moon could also result in a nearly sinusoidal TTV signature, but on a timescale this is typically much shorter than $\tau_{\text {slow }}$ (due to dynamical stability constraints). TTV perturbations with a timescale near $\tau_{\text {slow }}$ and due to non-resonant planets will have small amplitude, unless the outer planet is quite massive and potentially detectable by other methods. Therefore, we suggest that a large amplitude sinusoidal signal with a period near $\tau_{\text {slow }}$ might allow Trojans to be uniquely identified. We suggest future investigations to test this conjecture.

We caution that the TTV signature of an extrasolar Trojan could also be non-sinusoidal. For example, Trojans with large libration amplitudes can become significantly non-sinusoidal (reducing the rms TTV by upto $\sim 40 \%$ ). A Trojan planet in a horseshoe-shaped orbit would produce much larger TTV perturbations with a very different shape. If the primary and Trojan planets have different eccentricities, then there will be additional longer term periodicities in the TTV signal due to secular perturbations (Fig. 1, right). If there are Trojans at both L4 and L5, then the transit timing signature could be approximated as the sum of two such signals (similar frequency, but different amplitudes and phases). Similarly, swarms of Trojan companions librating about L4 and L5 could be modeled as the superposition of many such signals, provided that their mutual interactions are negligible. Additional planets could also perturb the time of central transit (Holman \& Murray 2005; Agol et al. 2005) such that the offset will vary from transit to transit. Therefore, many transits should be observed to verify that any observed offsets are not due to perturbations by a more distant giant planet.

The interpretation of actual TTV observations will be further complicated by constrained sampling (observations only possible during transit), incomplete sampling (due to available telescope time, and weather; Agol \& Steffen 2006) and measurement errors, all of which increases the uncertainties in the number, masses and orbits of planets. These limi- 
tations underscore the need for powerful statistical methods to interpret TTV observations. In cases where multiple orbital models are consistent with TTV data, additional observational constraints (e.g., radial velocities, secondary transit, changing transit duration due to

inclination librations) could help identify the correct model. We encourage further research in such methods, so that ongoing TTV observations can be appropriately analyzed.

We thank Eric Agol, Dan Fabrycky, Scott Gaudi, Jason Steffen, and Josh Winn, for helpful comments. Support for EBF was provided by NASA through Hubble Fellowship grant HST-HF-01195.01A awarded by the Space Telescope Science Institute, which is operated by the Association of Universities for Research in Astronomy, Inc., for NASA, under contract NAS 5-26555. MJH acknowledges support for this work NASA Origins grant NG06GH69G.

\section{REFERENCES}

Agol, E., Steffen, J., Sari, R., \& Clarkson, W. 2005, MNRAS, 359, 567

Agol, E, Steffen, J.H. 2007, MNRAS, 374, 941.

Bozorgnia, N., Fortney, J. J., McCarthy, C., Fischer, D. A., \& Marcy, G. W. 2006, PASP, 118,1252

Brown, T. M., Charbonneau, D., Gilliland, R. L., Noyes, R. W., \& Burrows, A. 2001, ApJ, 552,699

Butler, R. P., et al. 2006, ApJ, 646, 505

Charbonneau, D., Brown, T. M., Noyes, R. W., \& Gilliland, R. L. 2002, ApJ, 568, 377

Charbonneau, D., et al. 2006, ApJ, 636, 445

Chiang, E. I., \& Lithwick, Y. 2005, ApJ, 628, 520

Cresswell, P., \& Nelson, R. P. 2006, A\&A, 450, 833

Cumming, A. 2004, MNRAS, 354, 1165

Deming, D., Seager, S., Richardson, L. J., \& Harrington, J. 2005, Nature, 434, 740

Ford, E. B., Joshi, K. J., Rasio, F. A., \& Zbarsky, B. 2000, ApJ, 528, 336

Ford, E. B., Seager, S., \& Turner, E. L. 2001, Nature, 412, 885 
Ford, E. B., \& Gaudi, B. S. 2006, ApJ, 652, L137

Ford, E. B., \& Rasio, F. A. 2006, ApJ, 638, L45

Ford, E. B., \& Chiang, E. I. 2007, ApJ, in press astro-ph/0701745

Gaidos, E., Moskovitz, N., \& Williams, D. M. 2006, IAU Colloq. 200: Direct Imaging of Exoplanets: Science \& Techniques, 153

Gaudi, B. S. 2003, preprint (astro-ph/0307280)

Gaudi, B. S., \& Winn, J. N. 2006, preprint (astro-ph/0608071)

Goździewski, K., \& Konacki, M. 2006, ApJ, 647, 573

Harrington, J., Hansen, B. M., Luszcz, S. H., Seager, S., Deming, D., Menou, K., Cho, J. Y.-K., \& Richardson, L. J. 2006, Science, 314, 623

Heyl, J. S., \& Gladman, B. J. 2006, ArXiv Astrophysics e-prints, arXiv:astro-ph/0610267

Holman, M. J., \& Murray, N. W. 2005, Science, 307, 1288

Holman, M. J., et al. 2006, ApJ, 652, 1715

Konacki, M., \& Maciejewski, A. J. 1999, ApJ, 518, 442

Laughlin, G., \& Chambers, J. E. 2002, AJ, 124, 592

Lee, M. H., \& Peale, S. J. 2002, ApJ, 567, 596

Malhotra, R. 1993, ApJ, 407, 266

Michtchenko, T. A., Beaugé, C., \& Roig, F. 2001, AJ, 122, 3485

Miralda-Escudé, J. 2002, ApJ, 564, 1019

Morbidelli, A., Levison, H. F., Tsiganis, K., \& Gomes, R. 2005, Nature, 435, 462

Murray, C. D., \& Dermott, S. F. 2000, Solar System Dynamics, Cambridge, UK: Cambridge University Press.

Rasio, F. A., \& Ford, E. B. 1996, Science, 274, 954

Rasio, F. A., Nicholson, P. D., Shapiro, S. L., \& Teukolsky, S. A. 1992, Nature, 355, 325

Rowe, J. F., et al. 2006, ApJ, 646, 1241 
Sahu, K. C., et al. 2006, Nature, 443, 534

Schwarz, R., Pilat-Lohinger, E., Dvorak, R., Érdi, B., \& Sándor, Z. 2005, Astrobiology, 5, 579

Steffen, J.H. \& Agol, E. 2005, MNRAS, 364, L96.

Thommes, E. W. 2005, ApJ, 626, 1033

Valencia, D., Sasselov, D.D., O’Connell, R.J. 2007, ApJ, 656, 545

Winn, J.N., Holman M.J., Roussanova, A. 2007, ApJ, in press astro-ph/0611404

Wu, Y., \& Murray, N. 2003, ApJ, 589, 605 

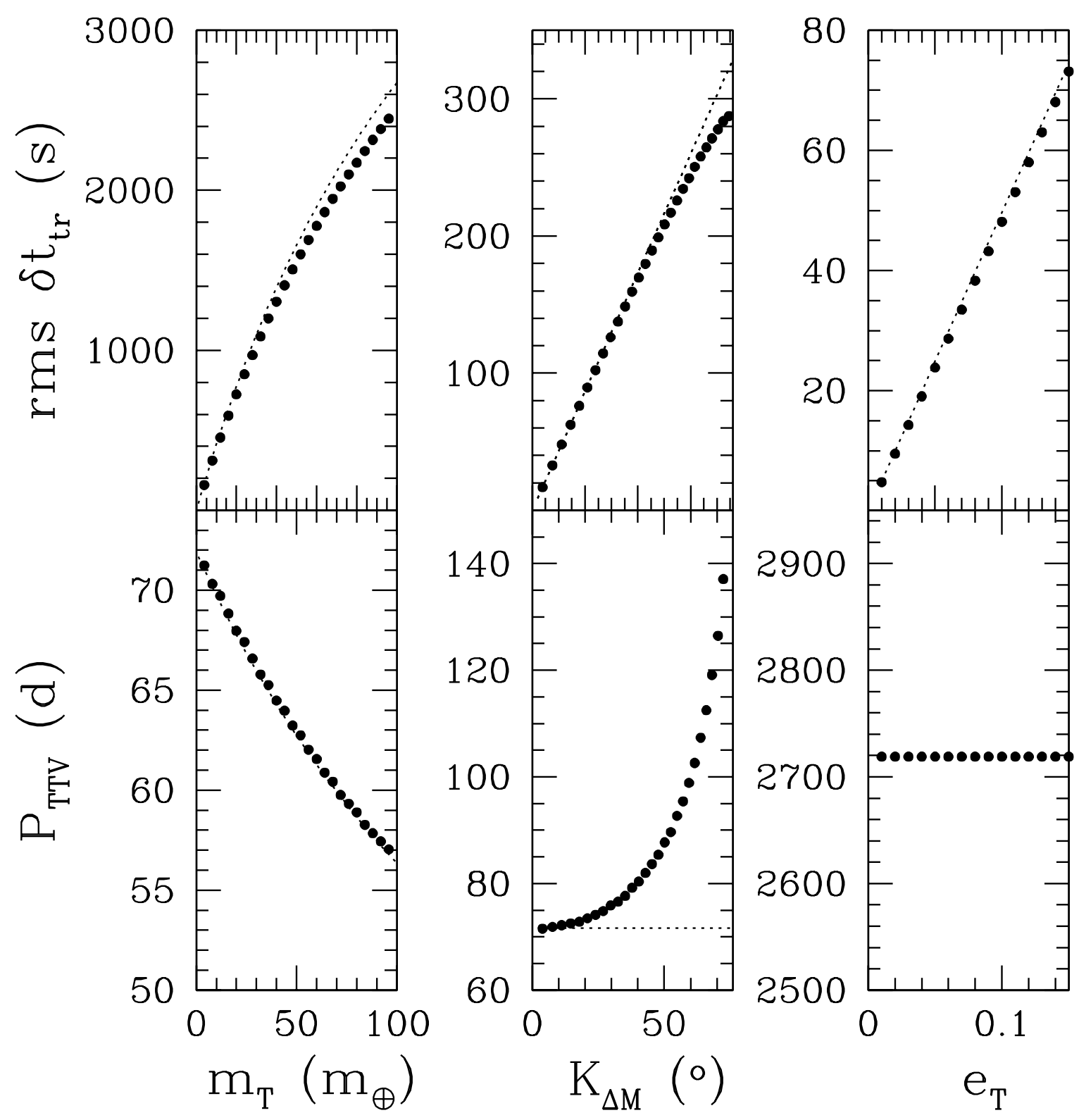

Fig. 1.- Transit Timing Signatures: We show the root mean square deviations of the TTVs from a strict periodicity (top) and the period of the transit timing variations (bottom) based on direct n-body integrations of a system with a $0.5 M_{\text {Jup }}$ planet and a Trojan orbiting a $M_{\odot}$ star. Dotted lines are analytic expressions from $\S 2$. (Left) The planet and the Trojan companion with mass $m_{T}$ are initially placed on circular orbits with a mean orbital separation of $0.05 \mathrm{AU}$ and $\Delta M_{T}=10^{\circ}$. (Center) As before, but as a function of $K_{\Delta M}$, the amplitude of the angular displacement from the Lagrange point, for a fixed Trojan mass of $1 M_{\oplus}$. (Right) As before, but as a function of initial eccentricity of the Trojan for a fixed Trojan mass of $1 M_{\oplus}$ and initial $\Delta M_{T}=0^{\circ}$. The dotted curve shows the analytic model, $\operatorname{rms}\left(\delta t_{t r}\right)=\epsilon P_{s} e_{T} /(\pi \sqrt{2})$. 


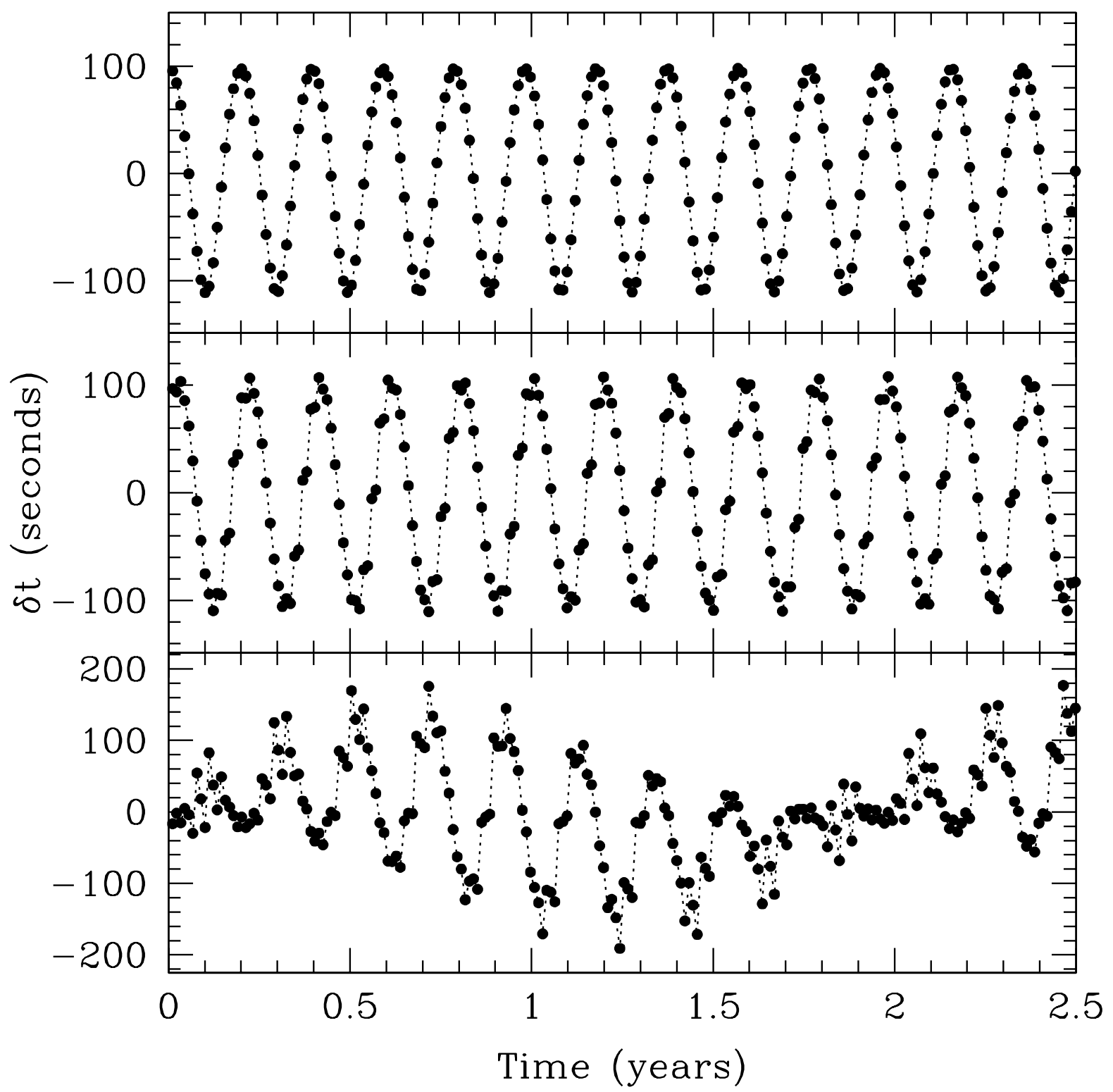

Fig. 2.- Similar Transit Timing Signatures due to Very Different Perturbing Planets: We plot the TTV residuals (disks) versus time for three hypothetical planetary systems. (The dotted lines merely guide the eye.) Each contains a typical transiting giant planet (0.5 Jupiter masses, orbital period of 4.09days) and a second planet. The perturbations are due to: top) a $1 M_{\oplus}$ Trojan companion, middle) the perturbations are due to a $28 M_{\oplus}$ (or 0.3 Saturn-mass) planet with a period of $\simeq 8$.7days (outside the 2:1 mean-motion resonance), and bottom) $\mathrm{a} \simeq 4.8$ Earth-mass planet with a period of $\simeq$ 5.91days (inside the 3:2 mean-motion resonance). Each planetary systems results in a TTV signature that has a dominant periodicity of 71.40days and a root-mean-square amplitude of 73.4s (based 1000 transits). Thus, interpreting TTV observations will require combining dynamical analyses with advanced statistical methods. 